Clinical and genetic characterization of patients with hypertrophic cardiomyopathy and right atrial enlargement

Giuseppe Limongelli, ${ }^{\mathrm{a} D a n i e l e M a s a r o n e},{ }^{\mathrm{a}}$ GiuliaFrisso, ${ }^{\mathrm{b}, \mathrm{c}}$ MariaIacomino, ${ }^{\mathrm{a}}$ Ilaria Ferrara, ${ }^{\mathrm{d}}$ Alessandra

Rea, ${ }^{\mathrm{a}}$ Rita Gravino, ${ }^{\mathrm{a} E d u a r d o}$ Bossone ${ }^{\mathrm{f}}$, Francesco Salvatore, ${ }^{\mathrm{b}}$ Raffaele Calabro', ${ }^{\text {a PerryElliott }}{ }^{\mathrm{e}}$, Giuseppe Pacileo ${ }^{\mathrm{a}}$

a Seconda Università degli Studi di Napoli, U.O.C. di Cardiologia, A.O. Monadi, Azienda dei Colli, Napoli, Italia

${ }^{\mathrm{b}}$ CEINGE-Biotecnologie Avanzate s.c.ar.1., Napoli, Italia

c Università degli Studi di Napoli “Federico II”, Dipartimento di Biochimica e Biotecnologie Mediche, Napoli, Italia

d Unità di “Imaging Cardiovascolare”, Ospedale Santa Maria delle Grazie, Pozzuoli, Italia

e Inherited Heart Disease Unit, The Hear Hospital, London, UK

${ }^{\mathrm{f}}$ Cardiology Division, "Cava de' Tirreni and Amalfi Coast" Hospital, Heart Department, University of Salerno; Cardiac Surgery Department, IRCCS Policlinico San Donato, Milan, Italy

Word Count:3216

Running Head: Right atrial enlargement in HCM

Corresponding Author:

Giuseppe Limongelli, MD, PhD, FESC, FAHA

Department of Cardiology

Monaldi Hospital

Second University of Naples

Naples, Italy

Tel: +390817062852

Fax: +390817062683

e-mail address: limongelligiuseppe@ libero.it 
Abstract

Aims: Prevalence and clinical significance of right atrial enlargement has been poorly characterized in hypertrophic cardiomyopathy

Methods: One hundred and sixty consecutively patients with hypertrophic cardiomyopathy (35.5 \pm 20 years; $64 \%$ men) were studied. They underwent clinical examination, standard ECG, Mmode, 2D and Doppler echocardiography, stress test and ECG Holter monitoring. Major adverse cardiac events were considered: 1) cardiac death (sudden death, heart failure death); 2) cardiac transplant; 3) resuscitated cardiac arrest or appropriate ICD discharge. Genetic analysis of 8 sarcomeric genes was performed using Sanger sequencing.

Results: Right atrial enlargement was observed in 22 patients (14\%), associated with left atrial enlargement in all cases. Patients with right atrial enlargement were likely to have restrictive mitral pattern $(\mathrm{p}<0.001)$ and had higher NYHA $(\mathrm{p}<0.001)$, pro NT BNP $(\mathrm{p}<0.001)$, left atrial volume in$\operatorname{dex}(\mathrm{p}<0.001)$, lateral $(\mathrm{p}=0.04)$ and septal $(\mathrm{p}=0.002) \mathrm{E} / \mathrm{e}$, systolic pulmonary artery pressure $(\mathrm{p}<0.001)$ and lower ejection fraction (all $\mathrm{p}<0.001)$. On cardiopulmonary exercise testing, peak VO2 was lower and VE/VCO2 higher in patients with right atrial enlargement $(\mathrm{p}<0.001)$.

During a mean follow-up of $4 \pm 2.1$ years, 30 MACE in 24 patients $(15 \%)$ were observed. Cox proportional hazards regression analysis, identified right atrial enlargement as an independent predictor of MACE $(\mathrm{OR}=2.6$; $\mathrm{CI} 1.5$ to 4.6; $\mathrm{p}=0.001)$. In patients with right atrial enlargement who were genetically tested, there was a higher prevalence of sarcomeric gene mutations (68\%) double mutations (16\%), and troponin T mutations (21\%).

Conclusions: RAE is present in a small subset of patients with HCM, and largely reflects increased pulmonary pressures due to severe diastolic and/or systolic LV dysfunction. Patients with RAE had a higher prevalence of sarcomeric gene mutations, troponin T mutations, and complex genotypes. In conclusion, RAE may serve as a very useful marker of disease progression and adverse outcome in patients with sarcomeric HCM. 
Keywords: hypertrophic cardiomyopathy, right atrial enlargement, sarcomeric gene mutation 
Introduction

Hypertrophic cardiomyopathy (HCM) is the most common inherited cardiac disorder, affecting approximately 1:500 subjects in the general population (1-3). The clinical expression of HCM is heterogeneous, and the awareness of the diverse disease spectrum and clinical course has expanded substantially over the last decade (4-5). Most individuals with HCM remain asymptomatic, but someone can experience progressive exercise intolerance and heart failure symptoms, and unexpected sudden cardiac death can occur at any age (6-8).

Left atrial enlargement (LAE) has been shown to be a sensitive marker of filling pressures and adverse prognosis in HCM (9-10). Right atrial enlargement (RAE) have been less well investigated in HCM. In this study, we sought to characterize prevalence, clinical significance and genetic epidemiology of RAE in HCM. We hypothesized that RAE represent a marker of disease severity, and thus measurement of right atrial size by conventional echocardiography may provide additional prognostic information in patients with HCM.

Methods

\section{Patient population}

This is an observational cross-sectional cohort study, including HCM patients seen from 2001 to 2011. All patients were from southern Italy and were of Caucasian descent. HCM was defined as unexplained left ventricular hypertrophy (wall thickness of $>15 \mathrm{~mm}$ in adults; $\mathrm{z}$-score value $>2$ in children). A previously described, syndromic $(n=34)$, metabolic $(n=4)$, infiltrative $(n=2)$ and neuromuscular $(\mathrm{n}=5)$ disorders associated with HCM were excluded following a standard protocol using non invasive, and when necessary, invasive investigations (muscle biopsy, fat/rectal biopsy and endomyocardial biopsy) (11). 


\section{Study protocol}

All patients underwent a complete cardiovascular evaluation, including family and personal history, physical examination, blood tests including $\mathrm{N}$-terminal prohormone of brain natriuretic peptide (NT-proBNP) measurement, 12-lead electrocardiogram (ECG) at rest, conventional M-mode, twodimensional and Doppler echocardiography, 24-hour Holter ECG and treadmill or cardiopulmonary exercise testing. New York Heart Association (NYHA) class based on the degree of dyspnoea or exercise limitation, was evaluated to define the severity of heart failure. Non sustained ventricular tachycardia during 24 hour ambulatory ECG monitoring was defined as three or more consecutive ventricular beats at rate of $\geq 120$ beats/min, lasting for $<30$ seconds. An abnormal blood pressure response during exercise was defined as initial increase in systolic blood pressure (SBP) with a subsequent fall of $>20 \mathrm{mmHg}$ compared with the blood pressure value at peak exercise or a continuous decrease in SBP throughout the exercise test of $>20 \mathrm{mmHg}$ compared with resting blood pressure or an increase of $<20 \mathrm{mmHg}$ in SBP from resting state to peak exercise (12). A clinical evaluation including standard ECG and echocardiography was generally repeated every six months. An ECG Holter and an exercise test were generally performed at least once a year. Genetic analysis was performed after informed consent, according to the procedure established by the Ethic Committee of our institution.

\section{Echocardiographic evaluation}

Left ventricular (LV) end-diastolic and end-systolic diameters and shortening fraction, end-diastolic thicknesses of interventricular septum and left ventricular posterior wall, were obtained from Mmode echocardiograms, according to recommendations of the American Society of Echocardiography (13).

At two-dimensional echocardiography, sites and maximal extent of ventricular hypertrophy were assessed in end-diastole by parasternal short axis view. LV end-diastolic and end-systolic volumes and ejection fraction $(\mathrm{EF})$ were calculated from apical views, using the biplane method of discs 
(modified Simpson's rule). An EF $<50 \%$ was considered abnormal. Left atrial (LA) volume index was measured using the biplane area-length method. The maximal LA area was measured in apical 4 and 2 chamber views by tracing the endocardial border, excluding the confluence of the pulmonary veins as well as the LA appendage. LA length was measured from the midline of the plane of the mitral annulus to the opposite superior aspect of the LA. The measurements were performed at end-systole from the frame immediately preceding mitral valve opening. LA volume greater than $34 \mathrm{ml} / \mathrm{m}^{2}$ was consider as LAE (14).

Right atrium (RA) area was calculated by planimetry (tracing the endocardium, and paying attention to exclude the possible redundancy of the great vein valves) in the apical four-chamber view at the point of tricuspid valve opening, and was corrected for height (RA area index), as previously reported (15). RA area greater than $18 \mathrm{~cm}^{2}$ was considered as RAE (16).

Systolic intraventricular gradients were assessed using continuous wave Doppler. A systolic peak gradient greater than $30 \mathrm{mmHg}$, at rest or after a Valsalva manouver, were considered abnormal.

LV diastolic function was assessed by pulsed Doppler at the level of mitral tips. Peak flow velocity in early diastole and during atrial contraction, and E/A ratio were measured, as well as deceleration time of early diastolic flow velocity. The pattern of left ventricular filling was classified as: restrictive if deceleration time $<120 \mathrm{~ms}$ or E/A $\geq 2$ associated with deceleration time $\leq 150 \mathrm{~ms}$; abnormal relaxation if E/A $<1$ associated with deceleration time $\geq 220 \mathrm{~ms}$; normal (or "pseudonormal") filling in presence of intermediate patterns (17). In patients with atrial fibrillation, only E wave and deceleration time were measured. Systolic, early diastolic (e'), and late diastolic tissue Doppler velocities were measured at the lateral mitral and septal walls and subsequently averaged over 3 cardiac cycles in accordance with previously reported methods (18). Transmitral E/e' ratios (lateral, septal, average) were calculated for each patient. E/e' average ratio was considered increased if higher than 15 . 


\section{Clinical Events}

Sudden cardiac death was defined as unexpected sudden collapse within one hour of onset of symptoms occurring in patients who had previously experienced a relatively stable or uneventful clinical course. Heart failure-related death was defined as death occurring in the context of cardiac decompensation and progressive disease course one year or more before death.

End stage evolution was defined as development of hypokinetic LV with an left ventricular EF $<50 \%$. In patients who had already presented at our center with a dilated and/or hypokinetic left ventricle, diagnosis of HCM always included previous echocardiographic documentation of classic HCM and family screening.

Major adverse cardiac events (MACE) were considered: 1) cardiac death (sudden death, death for heart failure); 2) cardiac transplant; 3) resuscitated cardiac arrest (“aborted sudden death") or appropriate implantable cardiac defibrillator discharge.

\section{Genetic analysis}

All exons, including the splicing sites, and 5' and 3' UTR regions of the beta myosin heavy chain, myosin binding protein $\mathrm{C}$, troponin $\mathrm{T}$, troponin $\mathrm{I}$, alpha tropomyosin, regulatory myosin light chain, essential myosin light chain, alpha-cardiac actin, protein kinase, AMP-activated, gamma 2 and lysosomal-associated membrane protein 2 were amplified by polymerase chain reaction, as previously described. Polymerase chain reaction products were then examined for sequence variations by Sanger sequencing. Nucleotide positions were numbered on the basis of the cDNA sequence according to the den Dunnen and Antonarakis nomenclature (19). When sequence variation was detected, the patient's available relatives underwent genetic screening.

\section{Statistical analysis}

Statistical analysis was performed using a commercially available package (SPSS, version15.0 2002, Chicago: SPSS Inc.). Data are presented as percentages, or means and standard deviations. Categorical variables were compared using the Fisher exact test or the $\chi^{2}$ statistic. Two groups of 
normally distributed variables were compared using the Student $t$ test, and analysis of variance was used to compare more than 2 groups. Skewed data were analyzed using the Mann Whitney U test, the Wilcoxon rank-sum test and the Kruskal-Wallis test, as appropriate. Linear regression and logistic, stepwise, multivariable models were performed to analyze predictors of RAE (baseline) and RA remodelling (during follow-up). For the survival analysis, the start of follow-up was defined as the first cardiovascular evaluation at Monaldi Hospital. Variables selected by univariate statistic $(\mathrm{p}<0.01)$ were selected for a multivariable Cox regression as an interactive stepwise backward elimination method, each time excluding the one variable with the highest p-value according to Wald statistics. The assumption of linearity was checked graphically by studying the smoothed martingale residuals from the null model plotted against the covariate variables. The linearity assumptions were satisfied. The Hosmer-Lemeshow goodness-of-fit test was used to check that the model adequately fit the data. In order to decrease the inflation of the Type 1 error rate due to multiple testing, the statistical significance was defined as two-sided p-value $<0.01$.

Results

\section{Study population}

The study cohort comprised one hundred and sixty consecutive patients. The mean age at the first evaluation was $35.5 \pm 20$ years (age ranging from birth to 74 years); 103 (64\%) patients were men. Family history of HCM was present in 84 patients (53\%), while family history of sudden cardiac death in 43 patients $(27 \%)$. The diagnosis was based on symptoms in 75 patients (47\%), incidental finding in 57 patients $(35.5 \%)$ and family screening in 28 patients $(17.5 \%)$.

RAE was observed in 22 patients $(13.7 \%)$, and was always associated with LAE (biatrial enlargement). Table 1 shows a comparison of clinical characteristics of patients with HCM with $(n=22)$ and without $(\mathrm{n}=138)$ RAE. Moderate to severe diastolic dysfunction and elevated non invasive wedge/systolic pulmonary pressure were observed in almost all patients with RAE, compared with no RAE patients $(\mathrm{p}<0.0001)$. Four patients $(18 \%)$ had an end stage disease (i.e. hypokinetic evolu- 
tion; $\mathrm{EF} \leq 50 \%$ ). Two patients had a cardiac arrest (at disease onset or in the first month after the first evaluation). A logistic, stepwise forward, multivariate model including all the significant variables at univariate analysis, demonstrated that systolic pulmonary artery pressure $\geq 30 \mathrm{mmHg}$ $(\mathrm{p}=0.025 ;$ OR $1.1 ; 95 \%$ CI 1-1.2) and NYHA class $\geq 2(\mathrm{p}=0.05 ;$ OR=3.9; 95\% CI 1.5-13) were independent predictors of RAE.

\section{Follow-up and cardiac events}

Clinical events are reported in Table 2. During a mean follow-up of $4 \pm 2.1$ years, a cardioverter defibrillator was implanted in 24 (15\%) and an appropriate discharge occurred in 3 patients (1.9\%). Seventeen patients experienced heart failure episodes requiring hospitalization, including 8 with RAE (5 patients with $\mathrm{EF}<50 \%)$ and 9 without $\mathrm{RAE}(7$ with $\mathrm{EF}<50 \%)$. Stroke events were more frequent in patients with $(14 \%)$ than patients without $(<1 \%)$ RAE. Four patients in the RAE cohort (18\%) were listed for transplant, 3 of them underwent successful orthotopic heart transplant, one patient underwent ventricular assist device implantation. Six patients, in the entire cohort, died. The cause of death was sudden in 1 patient in each group (HCM with or without RAE). Two patients, 1 from each group, died from complications soon after heart transplant surgery. The patient who underwent ventricular assist device implantation, died of multiorgan failure after 1 week. One patient with HCM without RAE died from non-cardiac causes. Cox proportional hazards regression analysis, with clinical and echocardiographic variables significant at chi-square analysis and univariate binomial model (including: $\mathrm{EF}<50 \%$, atrial fibrillation, restrictive filling pattern, septal E/e'>15) identified RAE (as a time-dependant covariate) as independent predictor of MACE (OR=2.6; 95\% CI 1.5, to 4.6; $\mathrm{p}=0.001$ ). Figure 1 shows a comparison of survival free of MACE between patients with RAE (RAE; $n=22 ; 14 \%)$, patients with isolated LAE $(n=101 ; 63 \%)$ and patients with no signs of atrial enlargement $(n=37 ; 23 \%)(p=0.0001)$. 


\section{Genetic results and genotype-phenotype correlation}

Genetic investigation has been completed for 94 of 160 patients with HCM: sequencing analysis of 10 genes identified a total of 61 mutations in 52 independent HCM patients (overall prevalence $55.3 \%)$. Of these, 59 (97\%) mutations have been previously reported as associated to HCM phenotype, whereas $2(3 \%)$ were novel variants. Novel mutations are absent from any publicly available databases (ESP and dbSNP137, that also includes the 1000 Genome Project data, MAF $=0$ ) and are considered pathogenetic by in silico functional prediction using bioinformatics programs (http://genetics.bwh.harvard.edu/pph/; $\quad$ http:// sift.jcvi.org/; http://neurocore. charite.de/MutationTaster, for coding variants; a specific software Alamut 2.0 was used to search for splicing prediction).

When we separated genotyped HCM patients with RAE $(n=20)$ from patients without RAE $(n=74)$ prevalence of sarcomeric mutations was significantly higher in the first group (85\% vs $50.3 \%$; $\mathrm{p}<0.004$, figure 3). Molecular epidemiology, with respect to 8 sarcomeric genes plus LAMP and PRKAG2, was different between the two group as regards TNNT2 mutations (20\% in HCM with RAE vs $3.7 \%$ in patients without RAE, p: 0.016). Overall, 8 patients (8.5\%) had a complex genotype, characterized by double-gene mutation heterozygosis $(n=4 ; 4.2 \%)$, compound heterozygosis $(\mathrm{n}=3 ; 3.2 \%)$, and homozygosis $(\mathrm{n}=1 ; 1 \%)$. Also complex genotypes were more frequent in HCM patients with RAE (20\% vs 5.3\% in patients without RAE, although it is not statistically different p: 0.058 - Figure 2).

\section{Discussion}

This study analyzed for the first time prevalence, clinical significance, and genetic background of RAE in a relatively large cohort of patients with HCM. The main findings of the study were: 1) the occurrence of RAE in a small subgroup (14\%) of HCM patients; 2) the association of RAE with MACE; 3) a high prevalence of sarcomeric mutations, TNNT2 mutations, and complex genotypes in patients with RAE. 


\section{Clinical Significance of RAE}

HCM is characterized by heterogeneous phenotypic expression. This affects the presence, degree and localization of myocardial hypertrophy, disarray, and fibrosis; the presence, severity and site of intracavitary obstruction; the presence and severity of diastolic and systolic dysfunction; and natural history and clinical outcome (20).

Heart failure symptoms in patients with HCM are mainly related to left ventricular outflow tract obstruction, diastolic dysfunction (heart failure with preserved ejection fraction), and systolic dysfunction (heart failure with reduced ejection fraction) (21). Left ventricular outflow tract obstruction, LAE, restrictive physiology and reduced EF $(<50 \%)$ are known to be associated with adverse prognosis in HCM (22-24). "Restrictive physiology" is generally evaluated by non invasive echocardiographic indices, such as transmitral and pulmonary flow velocities, and by using tissue Doppler measurements such as diastolic mitral annulus velocities (E/e' ratio). However, these indices shows only a modest correlation with the invasive measurement of left atrial and wedge pressure by cardiac catheterization, and their reproducibility is low in presence of atrial fibrillation and in the late stages of the disease (25). The measurement of right atrial size by conventional echocardiography is relatively easy and reproducible, even in presence of atrial fibrillation and in the end stage phase of the disease. However, the additional prognostic value of right atrial size measurements has not been analyzed, so far. In this study, patients with RAE were more symptomatic, had more severe exercise limitation, greater NT-proBNP elevation, elevated systolic pulmonary artery pressure, and presence of advanced diastolic and/or systolic dysfunction. In this study, RAE seems a good marker of end-stage progression, and largely reflects increased pulmonary pressures due to severe LV dysfunction (which may be purely diastolic or systo-diastolic). Thus, RAE may serve as a very useful marker of disease progression towards the end-stage phase. In clinical practice, RAE is generally considered a surrogate of severe restrictive physiology and pulmonary hypertension. However, a subset of patients had only mild diastolic abnormalities, suggesting that RAE may represent a relatively early marker 
of a "restrictive phenotype", preceding a clinically evident "restrictive physiology". Thus, measurement of RA size may be of help to guide clinical management of patients with non obstructive HCM, particularly when evaluation of systolic and diastolic function is complicated by atrial fibrillation.

Supportive evidence for this comes from a postmortem study by Melacini et al. looking at HCM patients who developed severe progressive heart failure. Interestingly, patients with non-obstructive HCM and RAE deteriorated to advanced NYHA classes III-IV associated with diastolic dysfunction or atrial fibrillation, compared to other HCM groups. Pathological examination of gross heart specimens showed small ventricular cavities, markedly enlarged atria, only focal scars of myocardial fibrosis, and mild left ventricular hypertrophy (26).

\section{Genetic Epidemiology and Pathogenesis of RAE}

The presence of RAE phenotype was associated with a higher frequency of mutations in sarcomeric genes (68\% vs. 43\%). A higher frequency of double mutations (16\% vs 0\%) was also observed. Recently, Biagini et al. studied a large cohort of end-stage HCM patients (27) and found complex genotypes with double or triple mutations in $13 \%$ compared with $5 \%$ of the reference cohort $(\mathrm{p}=0.01)$. However, as in the present study, the outcome of end stage HCM was independent of genotype. There was also a relatively high percentage of troponin $\mathrm{T}$ mutations. Of note, three patients with troponin T mutations had cardiac arrest (H11, H43, H133), while patient $\mathrm{H} 133$ has been listed for heart transplant. However, given the relatively small cohort of patients studied, it is was not possible to analyze a potential correlation between genotype and outcome in this subgroup.

The mechanism of RAE is unknown. Progressive diastolic dysfunction and atrial fibrillation may represent adequate substrates for right atrial remodeling. A progressive "atrial myopathy" has also been suggested in patients with HCM (28). A genetic basis of RAE is an attractive hypothesis, alt- 
hough it is likely that the atrial remodelling is a complex phenotype requiring both genetic (i.e. double mutations) and non genetic (haemodynamic) effects.

\section{Study Limitations}

Although our study population and the number of clinical events is relatively small, this is the first study to analyze prevalence and potential clinical impact of RAE in HCM. Recent guidelines recommend the use of RA volume as the gold standard to measure RA size (13). This study was conducted before 2015, and RA size was measured as suggested by previous guidelines (16). Only few patients underwent cardiac catheterization, with invasive estimation of left ventricular end-diastolic pressure and left/right atrial pressure measurements $(n=5)$. However, the correlation between the Doppler-derived diastolic parameters (transmitral Doppler flow velocities and mitral annular velocities) and invasive measurements measurement was behind the scope of the study .

\section{Conclusions}

RAE is present in a small subset of patients with HCM, and largely reflects increased pulmonary pressures due to severe diastolic and/or systolic LV dysfunction. Patients with RAE had a higher prevalence of sarcomeric gene mutations, troponin T mutations, and complex genotypes. In conclusion, RAE may serve as a very useful marker of disease progression and adverse outcome in patients with sarcomeric HCM. 


\section{REFERENCES}

1) Elliott PM, Anastasakis A, Borger MA, Borggrefe M, Cecchi F, Charron P et al. 2014 ESC Guidelines on diagnosis and management of hypertrophic cardiomyopathy: the Task Force for the Diagnosis and Management of Hypertrophic Cardiomyopathy of the European Society of Cardiology (ESC). Eur Heart J 2014 ;14:2733-79.

2) Gersh BJ, Maron BJ, Bonow RO, Dearani JA, Fifer MA, Link MS, et al. American College of Cardiology Foundation/American Heart Association Task Force on Practice Guidelines; American Association for ThoracicSurgery; American Society of Echocardiography; American Society of Nuclear Cardiology; HeartFailure Society of America; HeartRhythm Society; Society for Cardiovascular Angiography and Interventions; Society of Thoracic Surgeons.2011 ACCF/AHA guideline for the diagnosis and treatment of hypertrophic cardiomyopathy: a report of the American College of Cardiology Foundation/American Heart Association Task Force on Practice Guidelines. Circulation 2011 $13: 783-831$.

3) Maron BJ. Hypertrophic cardiomyopathy. A systematic review. JAMA 2002287:1308-1320.

4) Maron BJ, Casey SA, Hauser RG, Aeppli DM. Clinical course of hypertrophic cardiomyopathy with survival to advanced age. J Am Coll. Cardiol 2003;45:987-996

5) Takagi E, Yamakado T, Nakano T. Prognosis of completely asymptomatic adult patients with hypertrophic cardiomyopathy. J Am Coll Cardiol1999;33:206-211.

6) Biagini E, Coccolo F, Ferlito M, Perugini E, Rocchi G, Bacchi-Reggiani L, et al. Dilated-hypokinetic evolution of hypertrophic cardiomyopathy: prevalence, incidence, risk factors, and prognostic implications in pediatric and adult patients. J Am Coll Cardiol 2005;46:1543-1550. 
7) Harris KM, Spirito P, Maron MS, Zenovich AG, Formisano F, Lesser JR, et al. Prevalence, clinical profile, and significance of left ventricular remodeling in the end-stage phase of hypertrophic cardiomyopathy. Circulation 2006;114:216-225.

8) Maron BJ, Spirito P, Shen WK, Haas TS, Formisano F, Link MS, et al. Implantable cardioverter-defibrillators and prevention of sudden cardiac death in hypertrophic cardiomyopathy. JAMA 2007;298:405-412.

9) Tsang TS, Abhayaratna WP, Barnes ME, Miyasaka Y, Gersh BJ, Bailey KR, et al.

Prediction of cardiovascular outcomes with left atrial size: is volume superior to area or diameter? J Am Coll Cardiol 2006;47:1018-1023.

10) Nistri S, Olivotto I, Betocchi S, Losi MA, Valsecchi G, Pinamonti B, et al Prognostic significance of left atrial size in patients with hypertrophic cardiomyopathy (from the Italian Registry for Hypertrophic Cardiomyopathy). Am J Cardiol 2006;98:960 -965.

11) Rapezzi C, Arbustini E, Caforio AL, Charron P, Gimeno-Blanes J, HeliöT, et al. Diagnostic work-up in cardiomyopathies: bridging the gap between clinical phenotypes and final diagnosis. A position statement from the ESC Working Group on Myocardial and Pericardial Diseases. Eur Heart J 2013; 34:1448-1458

12) O'Mahony C1, Elliott P, McKenna W. Sudden cardiac death in hypertrophic cardiomyopathy. Circ Arrhythm Electrophysiol 2013;6:443-451

13) Lang RM, Badano LP, Mor-Avi V, Afilalo J, Armstrong A, Ernande L, et al Recommendations for cardiac chamber quantification by echocardiography in adults: an update from the American Society of Echocardiography and the European Association of Cardiovascular Imaging. J Am Soc Echocardiogr 2015;28:1-39

14) Aurigemma GP, Gottdiener JS, Arnold AM, Chinali M, Hill JC, Kitzman D. Left Atrial Volume and Geometry in Healthy Aging The Cardiovascular Health Study. Circ Cardiovasc Imaging 2009;2:282-289. 
15) D'Andrea A, Scarafile R, Riegler L, Salerno G, Gravino R, Cocchia R, et al. Right atrial size and deformation in patients with dilated cardiomyopathy undergoing cardiac resynchronization therapy. Eur J Heart Fail 2009; 11: 1169-1177

16) Rudski LG, Lai WW, Afilalo J, Hua L, Handschumacher MD, Chandrasekaran K, et al. Guidelines for the echocardiographic assessment of the right heart in adults: a report from the American Society of Echocardiography endorsed by the European Association of Echocardiography, a registered branch of the European Society of Cardiology, and the Canadian Society of Echocardiography. J Am Soc Echocardiogr 2010;23:685-713

17) Nagueh SF, Appleton CP, Gillebert TC, Marino PN, Oh JK, Smiseth OA, et al. Recommendations for the evaluation of left ventricular diastolic function by echocardiography. Eur J Echocardiogr 2009;10:165-193.

18) Geske JB, Sorajja P, Nishimura RA, Ommen SR. Evaluation of Left Ventricular Filling Pressures by Doppler Echocardiography in Patients With Hypertrophic Cardiomyopathy: Correlation With Direct Left Atrial Pressure Measurement at Cardiac Catheterization. Circulation 2007;116:2702-2708

19) den Dunnen JT, Antonarakis SE. Nomenclature for the description of human sequence variations. Hum Genet 2001;109:121-124.

20) Maron BJ, Ommen SR, Semsarian C, Spirito P, Olivotto I, Maron MS. Hypertrophic cardiomyopathy: present and future, with translation into contemporary cardiovascular medicine. J Am Coll Cardiol 2014;64:83-99.

21) Olivotto I, Cecchi F, Poggesi C, Yacoub M. Patterns of disease progression in hypertrophic cardiomyopathy. Circ Heart Fail. 2012;5:535-546

22) Losi MA, Betocchi S, Barbati G, Parisi V, Tocchetti CG, Pastore F et al. Prognostic significance of left atrial volume dilatation in patients with hypertrophic cardiomyopathy. $\mathrm{J}$ Am Soc Echocardiogr. 2009;22:76-81 
23) Biagini E, Spirito P, Rocchi G, Ferlito M, Rosmini S, Lai F, et al. Prognostic implications of the Doppler restrictive filling pattern in hypertrophic cardiomyopathy.Am J Cardiol 2009;104:1727-1731

24) Kawarai H, Kajimoto K, Minami Y, Hagiwara N, Kasanuki H. Risk of sudden death in endstage hypertrophic cardiomyopathy. J Card Fail 2011;17:459-464

25) Park JH, Marvick T. Use and Limitations of E/e' to Assess Left Ventricular Filling Pressure by Echocardiography.- Cardiovasc Ultrasound. 2011 Dec; 19: 169-173

26) Melacini P, Basso C, Angelini A, Calore C, Bobbo F, Tokajuk B, et al. Clinicopathological profiles of progressive heart failure in hypertrophic cardiomyopathy. European Heart Journal 2010; 31: 2111-2123.

27) Biagini E, Olivotto I, Iascone M, Parodi MI, Girolami F, Frisso G, et al.Significance of sarcomere gene mutations analysis in the end-stage phase of hypertrophic cardiomyopathy. Am J Cardiol 2014;114:769-776.

28) Olivotto I, Girolami F, Nistri S, Rossi A, Rega L, Garbini F, et al. The many faces of hypertrophic cardiomyopathy: from developmental biology to clinical practice. Cardiovasc Transl Res 2009;2:349-367. 


\section{Figure Legend}

Figure 1: Cumulative risk for adverse outcome in patients with classic HCM, patient with no atrial dilation, isolated LAE, and patients with RAE. MACE (major adverse cardiac events) was defined as combined end point including death, heart transplant/transplant list, aborted sudden death/appropriate ICD discharge.

Figure 2: Distribution of sarcomeric gene mutations in the study population: comparison between HCM patients with and without RAE. 


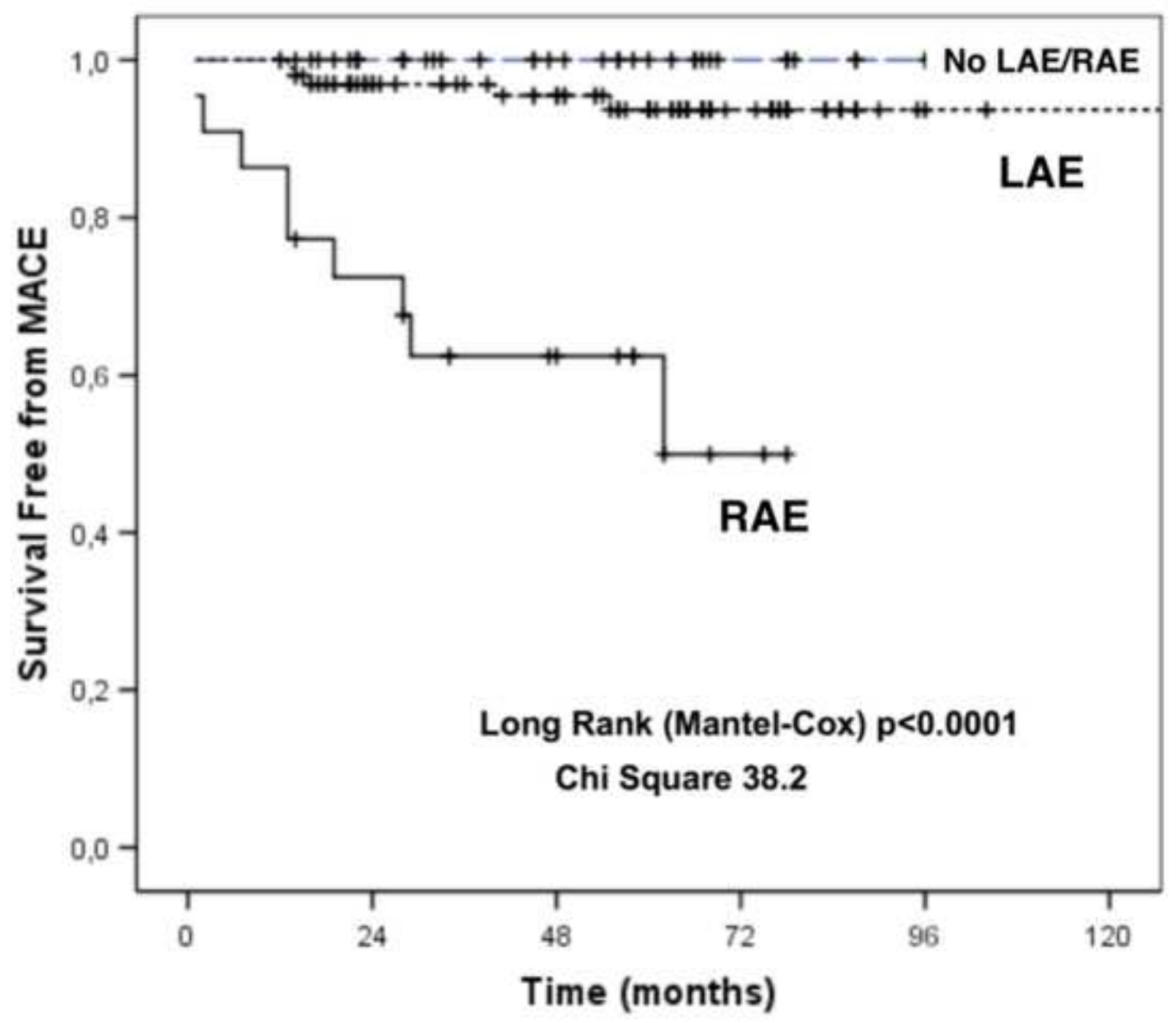




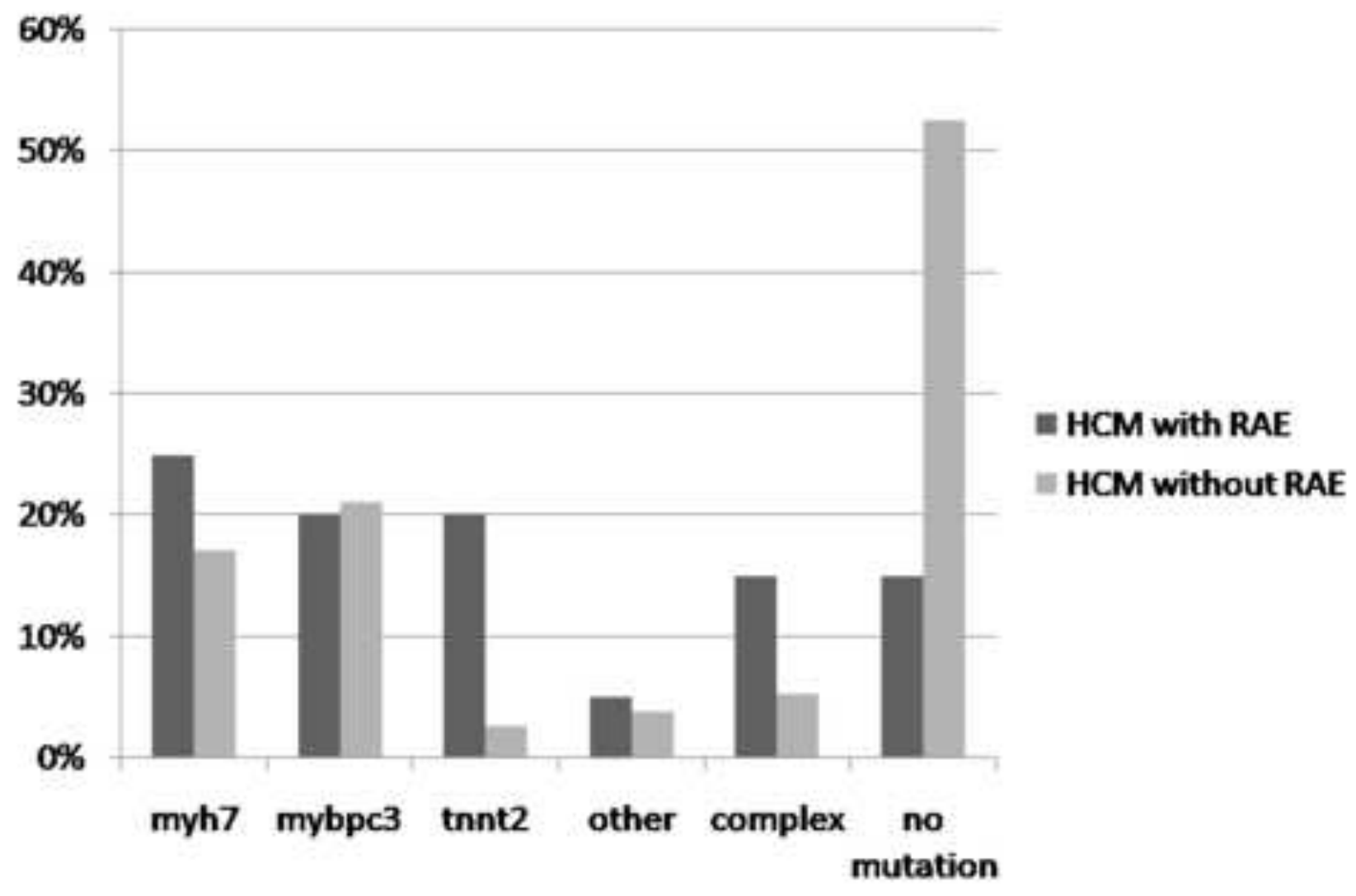


Table 1. Baseline characteristics of HCM patients with and without RAE at first evaluation.

\begin{tabular}{|c|c|c|c|}
\hline & $\begin{array}{l}\text { RAE } \\
(n=22)\end{array}$ & $\begin{array}{l}\text { No RAE } \\
(n=138)\end{array}$ & $p$ \\
\hline Family history of HCM $(\mathrm{n}, \%)$ & $10(45 \%)$ & $75(54 \%)$ & 0.44 \\
\hline Family history of SD (n,\%) & $7(32 \%)$ & $36(26 \%)$ & 0.57 \\
\hline Family history of ES $(\mathrm{n}, \%)$ & $6(27 \%)$ & $24(17 \%)$ & 0.27 \\
\hline Family history of RAE (n,\%) & $5(23 \%)$ & $5(3 \%)$ & $<0.001$ \\
\hline Age at diagnosis $(y)$ & $34 \pm 19$ & $28 \pm 20$ & 0.21 \\
\hline Age at first evaluation (y) & $39 \pm 18$ & $35 \pm 20$ & 0.41 \\
\hline $\operatorname{BSA}\left(\mathrm{cm}^{2}\right)$ & $1.8 \pm 0.3$ & $1.8 \pm 0.2$ & 0.77 \\
\hline NYHA Class & $2.5 \pm 0.4$ & $1.6 \pm 0.7$ & $<0.001$ \\
\hline Syncope (n,\%) & $5(23 \%)$ & $17(12 \%)$ & 0.19 \\
\hline Maximal wall thickness (mm) & $19.9 \pm 4.5$ & $20 \pm 5.8$ & 0.96 \\
\hline $\operatorname{LVEDD}(\mathrm{mm})$ & $49 \pm 6.7$ & $45 \pm 6.6$ & 0.009 \\
\hline $\operatorname{LAVI}\left(\mathrm{ml} / \mathrm{m}^{2}\right)$ & $61 \pm 27$ & $38 \pm 15$ & $<0.001$ \\
\hline RAA $\left(\mathrm{cm}^{2}\right)$ & $37 \pm 14$ & $18 \pm 2.2$ & $<0.001$ \\
\hline RAAI (cm2/m) & $18 \pm 9.5$ & $8 \pm 1.4$ & $<0.001$ \\
\hline TAPSE & $18.3 \pm 3.2$ & $19.3 \pm 2$ & 0.06 \\
\hline Moderate/Severe TR & $4(18 \%)$ & $(1 \%)$ & $<0.001$ \\
\hline $\operatorname{LVOT}(\mathrm{n}, \%)$ & $6(27 \%)$ & $46(33 \%)$ & 0.57 \\
\hline
\end{tabular}




\begin{tabular}{|c|c|c|c|}
\hline $\mathrm{EF}(\%)$ & $51 \pm 9$ & $58 \pm 7.7$ & $<0.001$ \\
\hline $\mathrm{E} / \mathrm{A}(\mathrm{m} / \mathrm{sec})$ & $1.83 \pm 0.8$ & $1.31 \pm 0.5$ & 0.001 \\
\hline MFP-Abnormal relaxation $(\mathrm{n}, \%)$ & $4(18 \%)$ & $96(69 \%)$ & $<0.001$ \\
\hline MFP-Pseudonormal $(\mathrm{n}, \%)$ & $12(54 \%)$ & $28(20 \%)$ & $<0.001$ \\
\hline MFP-Restrictive (n,\%) & $6(27 \%)$ & $2(18 \%)$ & $<0.001$ \\
\hline Septal E/e' ratio & $16.9 \pm 7.6$ & $12.4 \pm 6$ & 0.002 \\
\hline Lateral E/e' ratio & $11.6 \pm 4.4$ & $9.5 \pm 4.7$ & 0.046 \\
\hline Average E/e' ratio & $14 \pm 3.7$ & $10.8 \pm 2.5$ & $<0.001$ \\
\hline PAPs $(\mathrm{mmHg})$ & $44 \pm 13$ & $31 \pm 6.6$ & $<0.001$ \\
\hline NT proBNP $(\mathrm{pg} / \mathrm{ml})^{*}$ & $1609 \pm 1035$ & $545 \pm 893$ & $<0.001$ \\
\hline VO2 $(\mathrm{mg} / \mathrm{Kg} / \mathrm{min}) * *$ & $14.8 \pm 3.8$ & $19.9 \pm 5$ & $<0.001$ \\
\hline $\mathrm{VE} / \mathrm{VCO}_{2}$ slope $^{* * *}$ & $31.9 \pm 5.7$ & $27 \pm 6.8$ & $<0.001$ \\
\hline Atrial fibrillation $(\mathrm{n}, \%)$ & $11(50 \%)$ & $17(12 \%)$ & $<0.001$ \\
\hline $\operatorname{NSVT}(\mathrm{n}, \%)$ & $2(9 \%)$ & $5(4 \%)$ & 0.24 \\
\hline Medical treatment $(\mathrm{n}, \%)$ & $21(95 \%)$ & $109(79 \%)$ & 0.06 \\
\hline Diuretics $(\mathrm{n}, \%)$ & $12(55 \%)$ & $13(9 \%)$ & $<0.001$ \\
\hline Warfarin $(\mathrm{n}, \%)$ & $9(41 \%)$ & $10(7 \%)$ & $<0.001$ \\
\hline Beta Blockers (n,\%) & $15(68 \%)$ & $80(58 \%)$ & 0.36 \\
\hline
\end{tabular}




\begin{tabular}{|c|c|c|c|}
\hline Calciumantagonists (n,\%) & $3(14 \%)$ & $14(10 \%)$ & 0.62 \\
\hline Surgical Myectomy (n,\%) & $1(5 \%)$ & $5(4 \%)$ & 0.83 \\
\hline ICD implantation (n,\%) & $1(5 \%)$ & $1(0.7 \%)$ & $/$ \\
\hline Cardiac arrest (n,\%) & $2(9 \%)$ & $0(0 \%)$ & $/$ \\
\hline Heart failure episodes (n,\%) & $8(36 \%)$ & $6(4 \%)$ & $<0.001$ \\
\hline
\end{tabular}

BSA: body surface area; EF: ejection fraction; ES: end stage; HCM: hypertrophic cardiomyopathy; ICD: implantable cardioverter defibrillator; LAVI: left atrial volume index; LVEDD: left ventricular end-diastolic diameter; LVOT: left ventricular outflow tract obstruction; MFP: mitral filling pattern; NSVT: non-sustained ventricular tachycardia; NYHA: Hew York Heart Association; RAA: right atrial area; RAAI: right atrial area index; SD: sudden death; sPAP: systolic pulmonary artery pressure; TAPSE: Tricuspid annular plane systolic excursion; TR: tricuspid regurgitation.

*Available in 128 patients (HCM with BAE, $\mathrm{n}=22$ )

** Available in 98 patients (HCM with BAE, $\mathrm{n}=18$ )

*** Available in 89 patients (HCM with BAE, $\mathrm{n}=1$ 
Table 2. Cardiac events during follow-up.

\begin{tabular}{|c|c|c|c|}
\hline & $\begin{array}{l}\text { RAE } \\
(n=22)\end{array}$ & $\begin{array}{l}\text { No RAE } \\
(n=138)\end{array}$ & $p$ \\
\hline Surgical Myectomy $(\mathrm{n}, \%)$ & $1(5 \%)$ & $5(4 \%)$ & 0.83 \\
\hline Stroke $(\mathrm{n}, \%)$ & $3(14 \%)$ & $1(0.007 \%)$ & $<0.0001$ \\
\hline ICD implantation $(\mathrm{n}, \%)$ & $6(27.3 \%)$ & $18(13 \%)$ & 0.08 \\
\hline Heart failure episodes $(\mathrm{n}, \%)$ & $8(36.3 \%)$ & $9(6.5 \%)$ & $<0.0001$ \\
\hline Heart Transplant/VAD $(\mathrm{n}, \%)$ & $4(18 \%)$ & $1(0.7 \%)$ & $<0.0001$ \\
\hline $\begin{array}{c}\text { Appropriate ICD discharge } \\
(\mathrm{n}, \%)\end{array}$ & $1(4.5 \%)$ & $2(1 \%)$ & I \\
\hline Sudden Death $(\mathrm{n}, \%)$ & $1(4.5 \%)$ & $1(0.7 \%)$ & I \\
\hline Cardiac Death $(\mathrm{n}, \%)$ & $2(9 \%)$ & $1(0.7 \%)$ & I \\
\hline Non Cardiac Death $(\mathrm{n}, \%)$ & / & $1(0.7 \%)$ & I \\
\hline
\end{tabular}

ICD: implantable cardioverter defibrillator; RAE: right atrial enlargement, VAD: ventricular assist device. 